\title{
A New Approach for Estimating the Friction in Thin Film Lubrication
}

\author{
Dag Lukkassen, ${ }^{1}$ Annette Meidell, ${ }^{1}$ and Peter Wall ${ }^{2}$ \\ ${ }^{1}$ Narvik University College, 8505 Narvik, Norway \\ ${ }^{2}$ Department of Mathematics, Lulea University of Technology, 97187 Lulea, Sweden \\ Correspondence should be addressed to Peter Wall, wall@ltu.se
}

Received 10 September 2010; Accepted 11 December 2010

Academic Editor: K. Vajravelu

Copyright ( 2010 Dag Lukkassen et al. This is an open access article distributed under the Creative Commons Attribution License, which permits unrestricted use, distribution, and reproduction in any medium, provided the original work is properly cited.

\begin{abstract}
An important problem in the theory of lubrication is to model and analyze the effect of surface roughness on, for example, the friction and load carrying capacity. A direct numerical computation is often impossible since an extremely fine mesh is required to resolve the surface roughness. This suggests that one applies some averaging technique. The branch in mathematics which deals with this type of questions is known as homogenization. In this paper we present a completely new method for computing the friction. The main idea is that we study the variational problem corresponding to the Reynolds equation. We prove that the homogenized variational problem is closely related to the homogenized friction. Finally we use bounds on the homogenized Lagrangian to derive bounds for the friction. That these bounds can be used to efficiently compute the friction is demonstrated in a typical example.
\end{abstract}

\section{Introduction}

A fundamental problem in lubrication theory is to describe the flow behavior between two adjacent surfaces which are in relative motion. For example, this type of flow takes place in different kinds of bearings, hip joints, and gearboxes. The main unknown is often the pressure in the fluid. After computing the pressure it is possible to compute other fundamental quantities as the friction on the surfaces and the load carrying capacity. In this paper we develop a completely new technique to find the friction in problems, where the effects of surface roughness are taken into account.

Let the bearing domain, $\Omega$, be an open bounded subset of $\mathbb{R}^{2}$, and points in $\Omega$ are denoted by $x=\left(x_{1}, x_{2}\right)$. Let us assume that the lower surface is smooth and moving while 


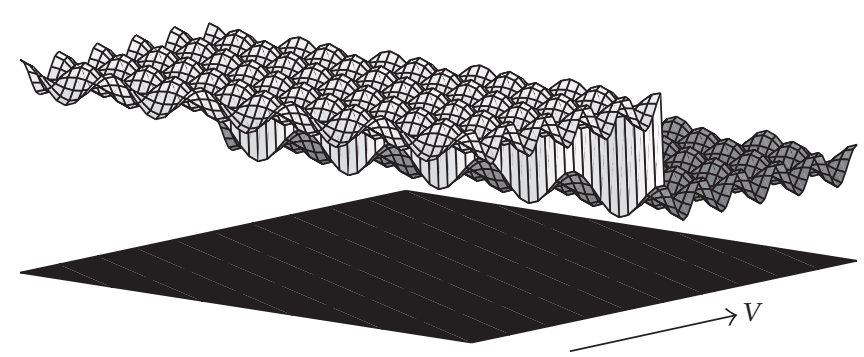

Figure 1: A smooth lower surface in motion and a rough stationary upper surface.

the upper surface is rough and stationary, see Figure 1 . The velocity of the upper surface is $V=\left(V_{1}, V_{2}\right)$. To express the film thickness we introduce the following auxiliary function:

$$
h(x, y)=h_{0}(x)+h_{r}(y)
$$

where $h_{0}$ and $h_{r}$ are smooth functions. Moreover, $h_{r}$ is $Y$-periodic, and we can without loss of generality assume that the cell of periodicity is the unit cube in $\mathbb{R}^{2}$, that is, $Y=(0,1) \times(0,1)$. By using the auxiliary function $h$ we can model the film thickness $h_{\varepsilon}$ by

$$
h_{\varepsilon}(x)=h\left(x, \frac{x}{\varepsilon}\right), \quad \varepsilon>0
$$

This means that $h_{0}$ represents the global film thickness, the periodic function $h_{r}$ describes the roughness on the upper surface, and $\varepsilon>0$ is a parameter which describes the wavelength of the roughness.

If the pressure is zero on the boundary, the fluid is Newtonian and has viscosity $\mu$, then the pressure due to the relative motions of the surfaces is modeled by the Reynolds equation, see, for example, [1] or [2]: find $p_{\varepsilon} \in W_{0}^{1,2}(\Omega)$ such that

$$
\operatorname{div}\left(h_{\varepsilon}^{3} \nabla p_{\varepsilon}\right)=6 \mu \operatorname{div}\left(h_{\varepsilon} V\right)
$$

On the surface $x_{3}=0$ the friction force, $F_{\varepsilon}$, is given by

$$
F_{\varepsilon}=\int_{\Omega} \frac{\mu}{h_{\varepsilon}} V+\frac{h_{\varepsilon}}{2} \nabla p_{\varepsilon} d x
$$

see, for example, [1].

In many applications the surfaces are rotating around the same axis. For example this is the situation in thrust pad bearings. Equation (1.3) in polar coordinates reads

$$
\frac{\partial}{\partial x_{1}}\left(\frac{h_{\varepsilon}^{3}}{x_{2}} \frac{\partial p_{\varepsilon}}{\partial x_{1}}\right)+\frac{\partial}{\partial x_{2}}\left(x_{2} h_{\varepsilon}^{3} \frac{\partial p_{\varepsilon}}{\partial x_{2}}\right)=6 \mu \omega x_{2} \operatorname{div}\left(h_{\varepsilon} e_{1}\right)
$$


where $\omega$ is the angular speed, $e_{1}=(1,0), x_{1}$ the angular coordinate, and $x_{2}$ is the radial coordinate. The friction torque is then

$$
\tau_{\varepsilon}=\int_{\Omega} \frac{\mu \omega x_{2}^{3}}{h_{\varepsilon}} d x+\int_{\Omega} \frac{h_{\varepsilon} x_{2}}{2} \frac{\partial p_{\varepsilon}}{\partial x_{1}} d x
$$

see, for example, [1].

We note that both (1.3) and (1.5) for the pressure $p_{\varepsilon} \in W_{0}^{1,2}(\Omega)$ are of the form

$$
\operatorname{div}\left(A_{\varepsilon} \nabla p_{\varepsilon}\right)=\operatorname{div}\left(b_{\varepsilon}\right)
$$

where

$$
A_{\varepsilon}(x)=\left(\begin{array}{cc}
a_{1}\left(x, \frac{x}{\varepsilon}\right) & 0 \\
0 & a_{2}\left(x, \frac{x}{\varepsilon}\right)
\end{array}\right), \quad b_{\varepsilon}(x)=\left(\begin{array}{c}
b_{1}\left(x, \frac{x}{\varepsilon}\right) \\
b_{2}\left(x, \frac{x}{\varepsilon}\right)
\end{array}\right) \text {. }
$$

For (1.3) we have $a_{i}(x, x / \varepsilon)=h_{\varepsilon}^{3}(x)$ and $b_{i}(x, x / \varepsilon)=6 \mu V_{i} h_{\varepsilon}(x)$ and for (1.5) $a_{1}(x, x / \varepsilon)=$ $h_{\varepsilon}^{3}(x) / x_{2}, a_{2}(x, x / \varepsilon)=x_{2} h_{\varepsilon}^{3}(x)$, and $b_{\varepsilon}(x)=6 \mu \omega x_{2} h_{\varepsilon}(x) e_{1}$.

A well-known fact from the calculus of variation is that the solution $p_{\varepsilon}$ of (1.7) also is the solution of the variational problem

$$
\min _{p \in W_{0}^{1,2}(\Omega)} I_{\varepsilon}(\nabla p)
$$

where

$$
I_{\mathcal{E}}(\nabla p)=\int_{\Omega}\left[\frac{1}{2} A_{\varepsilon} \nabla p \cdot \nabla p-b_{\varepsilon} \cdot \nabla p\right] d x
$$

That is, $I_{\varepsilon}\left(\nabla p_{\varepsilon}\right)=\min _{p} I_{\varepsilon}(\nabla p)$.

In order to compute $F_{\varepsilon}$ and $\tau_{\varepsilon}$ we have to find the partial derivatives of $p_{\varepsilon}$. However, this is a delicate problem. The main reason is that for small values of $\varepsilon$ (i.e., the roughness scale is much smaller than the global scale) the distance between the surfaces, $h_{\varepsilon}$, is rapidly oscillating. Thus a direct numerical treatment of (1.7) or (1.9) will require an extremely fine mesh to resolve the surface roughness. In many situations it is impossible even in practice. One approach is then to do some type of averaging. The field of mathematics which handles this type of averaging is known as homogenization, see, for example, [3] or [4]. Lately, homogenization has been used with success by many authors to study different types of the Reynolds equations. In particular, homogenization was applied to find the homogenized friction and friction torque in [5]. It should be mentioned that even though homogenization is used the numerical analysis is expensive since the homogenization procedure includes the solution of a number of local problems.

In this work we develop a new technique for estimating the homogenized friction. The main idea is that we first prove that the homogenized friction is closely related to the 
homogenized (averaged) variational problem corresponding to (1.9); secondly we apply our lower and upper bounds on the homogenized Lagrangian for (1.9) to obtain lower and upper bounds on the friction. As the bounds are close the mean value of the lower and upper bound will give a very accurate estimate of the friction. The benefit of the proposed method for estimating the homogenized friction, instead of computing it directly, is that no local partial differential equations have to be solved. This means that the new method requires a less computation time. We also illustrate the efficiency of the new method by applying it in a typical numerical example.

It should be mentioned that in the present paper the surface roughness is modelled as a periodic extension of a representative part of the roughness. This is crucial for the proposed method for computing the homogenized (averaged) friction. However, by using stochastic homogenization theory it is also possible to analyze related problems where the surface roughness is described as a realization of a stationary random field, see, for example, the book [4]. The first development of the stochastic theory, in the context of lubrication, was made in [6]. This work was limited to two-dimensional transverse and longitudinal roughness. Patir and Cheng were the first to propose a model for general roughness patterns, see $[7,8]$. Their derivation was heuristic, and it is now well known that it does not properly model situations where the roughness anisotropy directions are not identical to the Cartesian coordinate axes, see, for example, [9]. From a practical point of view the stochastic approach roughly leads to that one in the averaging process has to consider a number of realizations on a part of the roughness. The result from this averaging can also be obtained by the periodic approach if the representative part is chosen sufficiently large. In applications it is therefore sufficient to consider the surface as a periodic extension of a measured representative part. The results in this paper are based on bounds related to a known periodic roughness. In future works it would though be interesting to derive bounds for the homogenized Lagrangian in the case when only the distribution of the roughness is known and investigate if there is a connection with the friction.

\section{Homogenization}

The main idea in homogenization is to prove that there exists a $p_{0}$ such that $p_{\varepsilon} \rightarrow p_{0}$ as $\varepsilon \rightarrow 0$ and that $p_{0}$ solves a corresponding homogenized (averaged) problem, which does not involve any rapid oscillations. This means that $p_{0}$ may be used as an approximation of $p_{\varepsilon}$ for small values of $\varepsilon$. In this section we recall the main homogenization results concerning (1.7) and (1.9), for (1.7) see [10-14] and for (1.9) see [15].

Let $W_{\text {per }}^{1,2}(Y)$ be the closure of smooth $Y$-periodic functions with respect to the usual norm in $W^{1,2}(Y)$. Now we introduce the three local problems: find $w_{1}, w_{2}$, and $v$ in $L^{2}\left(\Omega ; W_{\text {per }}^{1,2}(Y)\right)$ such that

$$
\begin{gathered}
\operatorname{div}_{y}\left[A(x, y)\left(e_{i}+\nabla_{y} w_{i}(x, y)\right)\right]=0, \quad \text { on } Y, i=1,2, \\
\operatorname{div}_{y}[b(x, y)-A(x, y) \nabla v(x, y)]=0, \quad \text { on } Y .
\end{gathered}
$$

Here $e_{1}=(1,0)$ and $e_{2}=(0,1)$. Note that the domain in the local problems (2.1) is $Y$ and not $\Omega$. When the local solutions $w_{1}, w_{2}$, and $v$ are known they are used to define the 
homogenized matrix $A_{0}(x)$, the homogenized vector $b_{0}(x)$, and the homogenized scalar $c_{0}(x)$ in the following way:

$$
\begin{gathered}
A_{0} e_{i=} \int_{Y} A\left(e_{i}+\nabla_{y} w_{i}\right) d y, \quad i=1,2, \\
b_{0}=\int_{Y}\left(b-A \nabla_{y} v\right) d y, \\
c_{0}=\int_{Y} \frac{1}{2} A \nabla v \cdot \nabla v d y .
\end{gathered}
$$

It is now possible to define the homogenized integral functional $I_{0}$ as

$$
I_{0}(\nabla p)=\int_{\Omega} f_{0}(x, \nabla p) d x
$$

where the homogenized Lagrangian $f_{0}$ is given by

$$
f_{0}(x, \xi)=\frac{1}{2} A_{0}(x) \xi \cdot \xi-b_{0}(x) \cdot \xi-c_{0}(x)
$$

The solutions $p_{\varepsilon}$ of (1.9) converge weakly in $W_{0}^{1,2}(\Omega)$ to the solution $p_{0}$ of the homogenized variational problem

$$
\min _{p \in W_{0}^{1,2}(\Omega)} I_{0}(\nabla p)
$$

that is, $I_{0}\left(\nabla p_{0}\right)=\min _{p} I_{0}(\nabla p)$. Moreover, we have the following convergence:

$$
\lim _{\varepsilon \rightarrow 0} I_{\varepsilon}\left(\nabla p_{\varepsilon}\right)=I_{0}\left(\nabla p_{0}\right)=\int_{\Omega}\left(\frac{1}{2} A_{0}(x) \nabla p_{0} \cdot \nabla p_{0}-b_{0}(x) \cdot \nabla p_{0}-c_{0}(x)\right) d x
$$

We remark that $p_{0}$ also solves the Euler equation corresponding to (2.5), that is,

$$
\operatorname{div}\left(A_{0} \nabla p_{0}\right)=\operatorname{div} b_{0} \quad \text { in } \Omega
$$

\section{Bounds}

As mentioned in the introduction the new technique for computing the friction, which is developed in the present work, is based on our previous results concerning lower and upper bounds on the homogenized Lagrangian $f_{0}$ given in (2.4). The bounds were presented in [16], but for the reader's convenience we review the main results here. 
Let us start by introducing some functions which will appear in the formulation of the bounds. If either $(i, j)=(1,2)$ or $(i, j)=(2,1)$, then

$$
\begin{gathered}
a_{i}^{+}(x)=\left(\int_{0}^{1}\left(\int_{0}^{1} a_{i} d y_{j}\right)^{-1} d y_{i}\right)^{-1}, \quad a_{i}^{-}(x)=\int_{0}^{1}\left(\int_{0}^{1} \frac{1}{a_{i}} d y_{i}\right)^{-1} d y_{j}, \\
b_{i}^{+}(x)=a_{i}^{+}(x) \int_{0}^{1} \frac{\int_{0}^{1} b_{i} d y_{j}}{\int_{0}^{1} a_{i} d y_{j}} d y_{i}, \quad b_{i}^{-}=\int_{0}^{1}\left[\left(\int_{0}^{1} \frac{1}{a_{i}} d y_{i}\right)^{-1} \int_{0}^{1} \frac{b_{i}}{a_{i}} d y_{i}\right] d y_{j}, \\
c_{i}^{+}(x)=\frac{1}{2} a_{i}^{+}(x)\left(\int_{0}^{1} \frac{\int_{0}^{1} b_{i} d y_{j}}{a_{i} d y_{j}} d y_{i}\right)^{2}-\frac{1}{2} \int_{0}^{1} \frac{\left(\int_{0}^{1} b_{i} d y_{j}\right)^{2}}{\int_{0}^{1} a_{i} d y_{j}} d y_{i}, \\
c_{i}^{-}(x)=\frac{1}{2} \int_{0}^{1}\left[\left(\int_{0}^{1} \frac{1}{a_{i}} d y_{i}\right)^{-1}\left(\int_{0}^{1} \frac{b_{i}}{a_{i}} d y_{i}\right)^{2}\right] d y_{j}-\frac{1}{2} \int_{0}^{1} \int_{0}^{1} \frac{b_{i}^{2}}{a_{i}} d y_{i} d y_{j} .
\end{gathered}
$$

These functions are now used to define $f^{-}$and $f^{+}$as

$$
\begin{aligned}
& f^{-}(x, \xi)=\frac{1}{2} A^{-} \xi \cdot \xi-b^{-} \cdot \xi+c_{1}^{-}+c_{2}^{-}, \\
& f^{+}(x, \xi)=\frac{1}{2} A^{+} \xi \cdot \xi-b^{+} \cdot \xi+c_{1}^{+}+c_{2}^{+},
\end{aligned}
$$

where $\xi \in \mathbb{R}^{2}$,

$$
A^{ \pm}=\left(\begin{array}{cc}
a_{1}^{ \pm} & 0 \\
0 & a_{2}^{ \pm}
\end{array}\right), \quad b^{ \pm}=\left(\begin{array}{c}
b_{1}^{ \pm} \\
b_{2}^{ \pm}
\end{array}\right)
$$

The main result in [16] is that we have the following bounds on the homogenized Lagrangian $f_{0}$ :

$$
f^{-}(x, \xi) \leq f_{0}(x, \xi) \leq f^{+}(x, \xi)
$$

From this it is obvious that

$$
\min I^{-}(\nabla p) \leq \min I_{0}(\nabla p) \leq \min I^{+}(\nabla p),
$$

where the minimum is taken over all $p$ in $W_{0}^{1,2}(\Omega)$ and $I^{-}$and $I^{+}$are defined as

$$
\begin{aligned}
& I^{-}(\nabla p)=\int_{\Omega} f^{-}(x, \nabla p) d x, \\
& I^{+}(\nabla p)=\int_{\Omega} f^{+}(x, \nabla p) d x .
\end{aligned}
$$


If we use the notation $p^{-}$for the minimizer in the left hand side of (3.5) and $p^{+}$the minimizer in the right hand side of (3.5), that is,

$$
I^{-}\left(\nabla p^{-}\right)=\min _{p} I^{-}(\nabla p), \quad I^{+}\left(\nabla p^{+}\right) \min _{p} I^{+}(\nabla p),
$$

then (3.5) can be rewritten as

$$
I^{-}\left(\nabla p^{-}\right) \leq I_{0}\left(\nabla p_{0}\right) \leq I^{+}\left(\nabla p^{+}\right)
$$

This means that $I_{0}\left(\nabla p_{0}\right)$ can be estimated with high accuracy if the lower and upper bounds in (3.8) are close. This fact will be crucial later on.

The pressures $p^{-}$and $p^{+}$which are minimizers in the variational problems given in (3.7) can be found by solving the corresponding Euler equations. Indeed,

$$
\operatorname{div}\left(A^{ \pm} \nabla p^{ \pm}\right)=\operatorname{div}\left(b^{ \pm}\right) \quad \text { in } \Omega
$$

We remark that the bounds are optimal in the sense that there are surface roughnesses for which the bounds $f^{-}$and $f^{+}$coincide with $f_{0}$.

In $[17,18]$ it was shown by many numerical examples that the difference of the bounds solutions $p^{-}$and $p^{+}$is very small. Moreover, it was seen that the homogenized solution $p_{0}$ of (2.7) is between $p^{+}$and $p^{-}$. This together means that $\left(p^{+}+p^{-}\right) / 2$ can be used as a very good approximation of $p_{0}$. From a computational point of view this is useful since it is much easier to find $p^{+}$and $p^{-}$than $p_{0}$. The reason for this is that to be able to compute $p_{0}$ one first has to find $A_{0}$ and $b_{0}$ which involves the solutions of many local problems (parameterized in $x$ ), while one only has to integrate to find $A^{ \pm}$and $b^{ \pm}$, which are needed for the computation of $p^{ \pm}$. Thus it is clear that $\int_{\Omega}\left(p^{+}+p^{-}\right) / 2 d x$ may be used to compute the load carrying capacity. It is not obvious how $p^{+}$and $p^{-}$can be used to calculate the friction in different applications (if possible). The main result in this paper is that we prove how this can be done.

\section{Bounds for the Friction}

The physical interpretation of $I_{0}\left(\nabla p_{0}\right)$ has not been known. Hence the physical meaning of the estimates (3.8), that is, $I^{-}\left(\nabla p^{-}\right) \leq I_{0}\left(\nabla p_{0}\right) \leq I^{+}\left(\nabla p^{+}\right)$, is also unclear. However, in this section we will prove that $I_{0}\left(\nabla p_{0}\right)$ is closely related to the homogenized friction, which is induced by the relative motion. Moreover, we will show that we can obtain bounds for the friction via the bounds (3.8).

Let us consider the case with the Reynolds equation given in Cartesian coordinates, that is, when $a_{i}(x, x / \varepsilon)=h_{\varepsilon}^{3}(x)$ and $b_{i}(x, x / \varepsilon)=6 \mu V_{i} h_{\varepsilon}(x)$ ( $V_{i}$ is the constant speed in the $x_{i}$-direction). As mentioned in the introduction, the friction force, $F_{\varepsilon}$, on the surface $x_{3}=0$ is given by

$$
F_{\varepsilon}=\int_{\Omega} \frac{\mu}{h_{\varepsilon}} V+\frac{h_{\varepsilon}}{2} \nabla p_{\varepsilon} d x
$$


The component of the friction force in the direction $V$ of the motion is

$$
\mathcal{F}_{\varepsilon}=F_{\varepsilon} \cdot \frac{1}{|V|} V=\int_{\Omega} \frac{\mu|V|}{h_{\varepsilon}}+\frac{h_{\varepsilon}}{2|V|} V \cdot \nabla p_{\varepsilon} d x
$$

Let us now consider the generalized formulation of (1.7): find $p_{\varepsilon} \in W_{0}^{1,2}(\Omega)$ such that

$$
\int_{\Omega} A_{\varepsilon} \nabla p_{\varepsilon} \cdot \nabla \phi d x=\int_{\Omega} b_{\varepsilon} \cdot \nabla \phi d x
$$

for any $\phi \in W_{0}^{1,2}(\Omega)$. Formally this equation is obtained by first multiplying (1.7) by $\phi$ and thereafter using partial integration. In particular, for $\phi=p_{\varepsilon}$, it holds that

$$
\int_{\Omega} A_{\varepsilon} \nabla p_{\varepsilon} \cdot \nabla p_{\varepsilon} d x=\int_{\Omega} b_{\varepsilon} \cdot \nabla p_{\varepsilon} d x
$$

This implies that

$$
\begin{aligned}
I_{\varepsilon}\left(\nabla p_{\varepsilon}\right) & =\int_{\Omega} \frac{1}{2} A_{\varepsilon} \nabla p_{\varepsilon} \cdot \nabla p_{\varepsilon}-b_{\varepsilon} \cdot \nabla p_{\varepsilon} d x \\
& =-\int_{\Omega} \frac{1}{2} b_{\varepsilon} \cdot \nabla p_{\varepsilon} d x=-3 \mu \int_{\Omega} h_{\varepsilon} V \cdot \nabla p_{\varepsilon} d x .
\end{aligned}
$$

Thus

$$
\int_{\Omega} h_{\varepsilon} V \cdot \nabla p_{\varepsilon} d x=-\frac{1}{3 \mu} I_{\varepsilon}\left(\nabla p_{\varepsilon}\right)
$$

From (4.2) and (4.6) it follows that

$$
\mathcal{F}_{\varepsilon}=\mu|V| \int_{\Omega} \frac{1}{h_{\varepsilon}} d x-\frac{1}{6 \mu|V|} I_{\varepsilon}\left(\nabla p_{\varepsilon}\right)
$$

By using (2.6) it is possible to pass to the limit in this equality. We get

$$
\mathfrak{F}_{\varepsilon} \longrightarrow K-\frac{1}{6 \mu|V|} I_{0}\left(\nabla p_{0}\right):=\mathcal{F}_{0}
$$

where the constant $K$ is

$$
K=\mu|V| \int_{\Omega} \int_{Y} \frac{1}{h(x, y)} d y d x
$$


By (3.8) and (4.8) we get bounds on the homogenized (averaged) friction in the direction of the motion, $\mathcal{F}_{0}$,

$$
\mathcal{F}_{0}^{-} \leq \mathcal{F}_{0} \leq \mathcal{F}_{0}^{+}
$$

where

$$
\begin{aligned}
& \mathcal{F}_{0}^{-}=K-\frac{1}{6 \mu|V|} I^{+}\left(\nabla p^{+}\right), \\
& \mathcal{F}_{0}^{+}=K-\frac{1}{6 \mu|V|} I^{-}\left(\nabla p^{-}\right) .
\end{aligned}
$$

If the difference between $I^{+}\left(\nabla p^{+}\right)$and $I^{-}\left(\nabla p^{-}\right)$is small, then the average of $\mathcal{F}_{0}^{-}+\mathcal{F}_{0}^{+}$is a good approximation of the friction $\mathcal{F}_{0}$. The benefit of this approximation is that it is easier to compute $\mathcal{F}_{0}^{-}$and $\mathcal{F}_{0}^{+}$than $\mathcal{F}_{0}$. The reason for this is that in order to compute $\mathcal{F}_{0}$ we have to solve three local problems (parameterized in $x$ ), see $(2.1)$, but computation $\mathcal{F}_{0}^{-}$and $\mathcal{F}_{0}^{+}$does not involve solution of any local problems.

In order to calculate $\mathcal{F}_{0}^{-}$and $\mathcal{F}_{0}^{+}$we have to find $I^{+}\left(\nabla p^{+}\right)$and $I^{-}\left(\nabla p^{-}\right)$. Recall that

$$
\begin{aligned}
& I^{+}\left(\nabla p^{+}\right)=\int_{\Omega} \frac{1}{2} A^{+} \nabla p^{+} \cdot \nabla p^{+}-b^{+} \cdot \nabla p^{+}+c_{1}^{+}+c_{2}^{+} d x, \\
& I^{+}\left(\nabla p^{-}\right)=\int_{\Omega} \frac{1}{2} A^{-} \nabla p^{-} \cdot \nabla p^{-}-b^{-} \cdot \nabla p^{-}+c_{1}^{-}+c_{2}^{-} d x .
\end{aligned}
$$

From practical reasons it is sometimes a good idea to rewrite (4.12) and (4.13). Indeed, by choosing $p^{+}$as the test function in the generalized formulation of (3.9) we get that

$$
\int_{\Omega} A^{+} \nabla p^{+} \cdot \nabla p^{+} d x=\int_{\Omega} b^{+} \cdot \nabla p^{+} d x
$$

This implies that the expression (4.12) for $I^{+}\left(\nabla p^{+}\right)$can be rewritten as

$$
I^{+}\left(\nabla p^{+}\right)=\int_{\Omega} c_{1}^{+}+c_{2}^{+}-\frac{1}{2} b^{+} \cdot \nabla p^{+} d x
$$

In the same we get that

$$
I^{-}\left(\nabla p^{-}\right)=\int_{\Omega} c_{1}^{-}+c_{2}^{-}-\frac{1}{2} b^{-} \cdot \nabla p^{-} d x
$$

It should be mentioned that it is possible to pass to the limit in (4.2) directly. Indeed, it was proved in [14] that $\nabla p_{\varepsilon}$ two scale converges to $\nabla p_{0}+\nabla_{y} p_{1}$, where $p_{1}$ is of the form

$$
p_{1}(x, y)=w_{1}(x, y) \frac{\partial p_{0}}{\partial x_{1}}+w_{2}(x, y) \frac{\partial p_{0}}{\partial x_{2}}+v(x, y)
$$


For the friction we get

$$
\mathcal{F}_{\varepsilon} \rightarrow \int_{\Omega} \int_{Y} \frac{\mu|V|}{h}+\frac{h}{2|V|} V \cdot\left[\nabla p_{0}+\nabla_{y} p_{1}\right] d y d x
$$

We remark that the result obtained in this way agrees with [19], where the asymptotic behavior of pressure and stresses in a thin film flow with a rough boundary was analyzed by introducing two parameters corresponding to the film thickness and wavelength of the roughness. Also note that a direct asymptotic analysis of (4.2) leads to that $p_{0}, w_{1}, w_{2}$, and $v$ have to be found.

\section{Bounds for the Friction Torque}

In many applications the surfaces are rotating around the same axis. For example, this is the situation in thrust pad bearings. The governing equation for the pressure is then (1.5), that is, $a_{1}(x, x / \varepsilon)=h_{\varepsilon}^{3}(x) / x_{2}, a_{2}(x, x / \varepsilon)=x_{2} h_{\varepsilon}^{3}(x)$ and $b_{\varepsilon}(x)=6 \mu \omega x_{2} h_{\varepsilon}(x) e_{1}$. The friction torque is then given by (1.6), that is,

$$
\tau_{\varepsilon}=\int_{\Omega} \frac{\mu \omega x_{2}^{3}}{h_{\varepsilon}} d x+\int_{\Omega} \frac{h_{\varepsilon} x_{2}}{2} \frac{\partial p_{\varepsilon}}{\partial x_{1}} d x
$$

see, for example, [1]. In the same way as for the friction force we get that

$$
I_{\varepsilon}\left(\nabla p_{\varepsilon}\right)=-\int_{\Omega} \frac{1}{2} b_{\varepsilon} \cdot \nabla p_{\varepsilon} d x=-6 \mu \omega \int_{\Omega} \frac{h_{\varepsilon} x_{2}}{2} \frac{\partial p_{\varepsilon}}{\partial x_{1}} d x
$$

Combining (1.6) and (5.2) gives

$$
\tau_{\varepsilon}=\int_{\Omega} \frac{\mu \omega x_{2}^{3}}{h_{\varepsilon}} d x-\frac{1}{6 \mu \omega} I_{\varepsilon}\left(\nabla p_{\varepsilon}\right)
$$

In the limit

$$
\tau_{\varepsilon} \rightarrow C-\frac{1}{6 \mu \omega} I_{0}\left(\nabla p_{0}\right):=\tau_{0}
$$

where the constant $C$ is

$$
C=\mu \omega \int_{\Omega} \int_{Y} \frac{x_{2}^{3}}{h(x, y)} d y d x
$$

In a similar way, as for the friction in the previous section, we can obtain bounds on the friction torque from (3.8) and (5.4). Indeed,

$$
\tau_{0}^{-} \leq \tau_{0} \leq \tau_{0}^{+}
$$


Table 1: Table of input data for the numerical example.

\begin{tabular}{lccc}
\hline Parameter & Description & Value & Unit \\
\hline$L_{1}$ & Pad length $\left(x_{1}\right)$ & 0.1 & $\mathrm{~m}$ \\
$L_{2}$ & Pad length $\left(x_{2}\right)$ & 0.2 & $\mathrm{~m}$ \\
$\mu$ & Fluid viscosity & 0.2 & $\mathrm{~Pa} \mathrm{~s}$ \\
$A$ & Roughness amplitude & $0.4 \cdot 10^{-5}$ & $\mathrm{~m}$ \\
$h_{1}$ & Maximum of $h_{0}$ & $2.0 \cdot 10^{-5}$ & $\mathrm{~m}$ \\
$h_{2}$ & Minimum of $h_{0}$ & $1.0 \cdot 10^{-5}$ & $\mathrm{~m}$ \\
$V=\left(V_{1}, V_{2}\right)$ & Velocity of the upper surface & $(1,0)$ & $\mathrm{m} / \mathrm{s}$ \\
\hline
\end{tabular}

where

$$
\tau_{0}^{-}=C-\frac{1}{6 \mu \omega} I^{+}\left(\nabla p^{+}\right), \quad \tau_{0}^{+}=C-\frac{1}{6 \mu \omega} I^{-}\left(\nabla p^{-}\right)
$$

As for the friction this motivates that the average of $\tau_{0}^{-}$and $\tau_{0}^{+}$can be used to approximately find the homogenized friction torque $\tau_{0}$.

\section{A Numerical Example}

In this section we apply the new method for computing the friction in a typical application. Indeed, we will estimate the friction force in a step bearing with bicosinusoidal roughness. More precisely, we consider the case where $h_{0}$ and $h_{r}$ are of the form

$$
\begin{gathered}
h_{0}(x)= \begin{cases}h_{1}, & 0<x_{1} \leq \frac{L_{1}}{2}, 0<x_{2}<L_{2}, \\
h_{2}, & \frac{L_{1}}{2}<x_{1}<L_{1}, 0<x_{2}<L_{2},\end{cases} \\
h_{r}(y)=A \cos \left(2 \pi y_{1}\right) \cos \left(2 \pi y_{2}\right) .
\end{gathered}
$$

The data that is used is presented in Table 1. In the numerical analysis we used the software COMSOL and MATLAB.

It is natural to ask if the effect of the surface roughness is negligible or not. In order to give some answer to this question we consider the two extreme cases, where the distance between the surfaces is assumed to be $h_{\min }=h_{0}-A$ and $h_{\max }=h_{0}+A$ ( $A$ is the amplitude of the surface roughness). Denote the friction forces corresponding to $h_{\min }$ and $h_{\max }$ by $\mathcal{F}_{\text {min }}$ respective $\mathcal{F}_{\max }$. Since $h_{\min } \leq h_{\max }$ it is obvious that the homogenized friction force, $\mathcal{F}_{0}$, satisfies the estimates $\mathcal{F}_{\max } \leq \mathcal{F}_{0} \leq \mathcal{F}_{\min }$. In our example we get that $\mathcal{F}_{\min }=559.85 \mathrm{~N}$ and $\mathcal{F}_{\max }=252.61 \mathrm{~N}$. We also have that the friction force, $\mathcal{F}_{00}$, corresponding to that the distance between the surfaces is just $h_{0}$ (i.e., no roughness) is $\mathcal{F}_{00}=348.64 \mathrm{~N}$ (observe that the choice of roughness implies that $h_{0}$ equals $h_{0}$ plus the average of the roughness $h_{r}$ ). This indicates that the influence of the surface roughness on the friction is significant and thus has to be taken into account. 
Table 2: Table of input data for the numerical example.

\begin{tabular}{lcc}
\hline$h_{0}$ & $1.0000 \cdot 10^{-5}$ & $2.0000 \cdot 10^{-5}$ \\
$a_{1}^{+}\left(h_{0}\right)$ & $1.1136 \cdot 10^{-15}$ & $8.2365 \cdot 10^{-15}$ \\
$a_{1}^{-}\left(h_{0}\right)$ & $7.9000 \cdot 10^{-16}$ & $7.5359 \cdot 10^{-15}$ \\
$b_{1}^{+}\left(h_{0}\right)$ & $1.2000 \cdot 10^{-5}$ & $2.4000 \cdot 10^{-5}$ \\
$b_{1}^{-}\left(h_{0}\right)$ & $1.0641 \cdot 10^{-5}$ & $2.3291 \cdot 10^{-5}$ \\
$c_{1}^{+}\left(h_{0}\right)$ & 0 & 0 \\
$c_{1}^{-}\left(h_{0}\right)$ & $-2.8983 \cdot 10^{3}$ & $-3.6014 \cdot 10^{2}$ \\
\hline
\end{tabular}

We will now illustrate that the new proposed method, based on bounds, gives very good estimates of the friction. Let us first note that the upper surface only moves in the $x_{1}$ direction, that is, $V_{2}=0$. From this it follows that $b_{2}(x, x / \varepsilon)=0$, which in turn implies that $b_{2}^{ \pm}=0$ and $c_{2}^{ \pm}=0$. Due to symmetry it also holds that $a_{1}^{ \pm}=a_{2}^{ \pm}$. In Table 2 the coefficients for the bounds (3.2) are presented.

Moreover, the constant $K$ in the bounds in (4.10) for $\mathcal{F}_{0}^{-}$and $\mathcal{F}_{0}^{+}$is 309.83 . The bounds become

$$
\begin{aligned}
& \mathcal{F}_{0}^{-}=K+\frac{1}{6 \mu|V|} \int_{\Omega} \frac{1}{2} b^{+} \cdot \nabla p^{+}-c_{1}^{+} d x=356.65 \mathrm{~N} \\
& \mathscr{F}_{0}^{+}=K+\frac{1}{6 \mu|V|} \int_{\Omega} \frac{1}{2} b^{-} \cdot \nabla p^{-}-c_{1}^{-} d x=395.41 \mathrm{~N}
\end{aligned}
$$

The new method presented in this paper is that we use the average of $\mathcal{F}_{0}^{-}+\mathcal{F}_{0}^{+}$as an approximation of the friction. We get that

$$
\frac{\mathcal{F}_{0}^{-}+\mathscr{F}_{0}^{+}}{2}=376.03 \mathrm{~N}
$$

It should be observed that if we approximate the distance between the surfaces with $h_{0}$ plus the mean value of $h_{r}$ and thereafter compute the friction, then the friction (denoted by $\mathcal{F}_{00}$ ) is underestimated.

Let us also compare the average of $\mathcal{F}_{0}^{-}+\mathscr{F}_{0}^{+}$with the homogenized friction. Indeed, first we solve the local problems (2.1). Secondly, we compute the homogenized matrix $A_{0}(x)$, the homogenized vector $b_{0}(x)$, and the homogenized scalar $c_{0}(x)$ given by (2.2). We obtain that

$$
\begin{gathered}
c_{0}(x)=\left\{\begin{array}{c}
1.8141 \cdot 10^{2}, \quad 0<x_{1} \leq \frac{L_{1}}{2}, 0<x_{2} \leq L_{2}, \\
1.4912 \cdot 10^{3}, \quad \frac{L_{1}}{2}<x_{1} \leq L_{1}, 0<x_{2} \leq L_{2},
\end{array}\right. \\
A_{0}(x)=\left(\begin{array}{cc}
a_{0}(x) & 0 \\
0 & a_{0}(x)
\end{array}\right), \quad b_{0}(x)=\left(\begin{array}{c}
b_{0}^{1}(x) \\
0
\end{array}\right),
\end{gathered}
$$


where

$$
\begin{aligned}
& a_{0}(x)= \begin{cases}7.8836 \cdot 10^{-15}, & 0<x_{1} \leq \frac{L_{1}}{2}, 0<x_{2} \leq L_{2}, \\
9.4686 \cdot 10^{-16}, & \frac{L_{1}}{2}<x_{1} \leq L_{1}, 0<x_{2} \leq L_{2},\end{cases} \\
& b_{0}^{1}(x)= \begin{cases}2.3643 \cdot 10^{-5}, & 0<x_{1} \leq \frac{L_{1}}{2}, 0<x_{2} \leq L_{2}, \\
1.1301 \cdot 10^{-5}, & \frac{L_{1}}{2}<x_{1} \leq L_{1}, 0<x_{2} \leq L_{2} .\end{cases}
\end{aligned}
$$

After computing $p_{0}$ given by (2.7) we find that

$$
\begin{aligned}
I_{0}\left(\nabla p_{0}\right) & =\int_{\Omega}\left(\frac{1}{2} A_{0}(x) \nabla p_{0} \cdot \nabla p_{0}-b_{0}(x) \cdot \nabla p_{0}-c_{0}(x)\right) d x \\
& =-\int_{\Omega} \frac{1}{2} b_{0}(x) \cdot \nabla p_{0}+c_{0}(x) d x=-79.653
\end{aligned}
$$

which implies that

$$
\mathcal{F}_{0}=K-\frac{1}{6 \mu|V|} I_{0}\left(\nabla p_{0}\right)=376.21 \mathrm{~N}
$$

As mentioned before, homogenization can be used to find good approximate answers to various problems for small values of $\varepsilon$. This is due to various convergence results as $\varepsilon \rightarrow 0$. However, it is natural to ask how small $\varepsilon$ has to be in order to have a good approximation. It has been shown in many examples in previous works that homogenization applies for rather large values of $\varepsilon$, much larger than in most typical applications. In the present example we have that $\mathcal{F}_{0.01}=376.08 \mathrm{~N}, \mathcal{F}_{0.005}=376.15 \mathrm{~N}$, and $\mathcal{F}_{0.004}=376.17 \mathrm{~N}$.

\section{Concluding Remarks}

We have proposed a new method, which takes surface roughness into account, for computing the friction in, for example, different bearings. The main idea is that we use the variational formulation of the corresponding Reynolds equation. First we prove that the homogenized friction is closely related to the homogenized variational problem. Thereafter we use our previous results concerning bounds for the homogenized Lagrangian to obtain bounds for the homogenized friction. That the method is applicable is shown in a typical example.

In order not to hide the main ideas we have considered the case when only one surface is rough. If both surfaces are assumed to be rough, then the coefficients (which are related to the distance between the surfaces) in the variational problem will involve rapid oscillations not only in space but also in time. The homogenization in this case has been studied in, for example, $[15,20,21]$. Bounds for the homogenized Lagrangian follow in this case in the same way, see [17]. Taking this into account it is obvious that the ideas in the present work also apply in order to compute the friction when both surfaces are rough. In [18] less sharp bounds 
(Reuss-Voigt type) were used to compute the corresponding $p^{ \pm}$. These bounds are in general wider. However, they are easier to compute, but can still give sufficient information. Clearly, the results in this work imply that the Reuss-Voigt type bounds can be used to generate bounds for the homogenized friction.

\section{Acknowledgment}

The authors thank the reviewers for the helpful comments.

\section{References}

[1] B. Hamrock, Fundamentals of Fluid Film Lubrication, Mechanical Engineering, McGraw-Hill, New York, NY, USA, 1994.

[2] O. Reynolds, "On the theory of lubrication and its application to Mr. Beuchamp tower's experiments, including an experimental determination of the viscosity of olive oil," Philosophical Transactions of the Royal Society A, vol. 117, pp. 157-234, 1886.

[3] D. Cioranescu and P. Donato, An Introduction to Homogenization, vol. 17 of Oxford Lecture Series in Mathematics and its Applications, Oxford University Press, Oxford, Uk, 1999.

[4] V. V. Jikov, S. M. Kozlov, and O. A. Oleĭnik, Homogenization of Differential Operators and Integral Functionals, Springer, Berlin, Germany, 1994.

[5] A. Almqvist, E. K. Essel, J. Fabricius, and P. Wall, "Reiterated homogenization applied in hydrodynamic lubrication," Proceedings of the Institution of Mechanical Engineers, Part J: Journal of Engineering Tribology, vol. 222, no. 7, pp. 827-841, 2008.

[6] H. Christensen and K. Tønder, "Tribology of rough surfaces. Stochastic models of hydrodynamic lubrication," Tech. Rep. 18-69/10, SINTEF, Trondheim, Norway, 1969.

[7] N. Patir and H. S. Cheng, "An average flow model for determining effects of three-dimensional roughness on partial hydrodynamic lubrication," Journal of Lubrication Technology, vol. 100, no. 1, pp. 12-17, 1978.

[8] N. Patir and H. S. Cheng, "Application of average fow model to lubrication between rough sliding surfaces," Transactions ASME Journal of Lubrication Technology, vol. 101, no. 2, pp. 220-230, 1979.

[9] M. Prat, F. Plouraboué, and N. Letalleur, "Averaged Reynolds equation for flows between rough surfaces in sliding motion," Transport in Porous Media, vol. 48, no. 3, pp. 291-313, 2002.

[10] G. Bayada and M. Chambat, "Homogenization of the Stokes system in a thin film flow with rapidly varying thickness," Modélisation Mathématique et Analyse Numérique, vol. 23, no. 2, pp. 205-234, 1989.

[11] G. Bayada and J. B. Faure, "Double scale analysis approach of the Reynolds roughness comments and application to the journal bearing," Journal of Tribology, vol. 111, no. 2, pp. 323-330, 1989.

[12] M. Chambat, G. Bayada, and J. B. Faure, "Some effects of the boundary roughness in a thin film," in Boundary Control and Boundary Variations, vol. 100 of Lecture Notes in Computer Science, pp. 96-115, Springer, Berlin, Germany, 1988.

[13] M. Kane and B. Bou-Said, "Comparison of homogenization and direct techniques for the treatment of roughness in incompressible lubrication," Journal of Tribology, vol. 126, no. 4, pp. 733-737, 2004.

[14] Peter Wall, "Homogenization of Reynolds equation by two-scale convergence," Chinese Annals of Mathematics. Series B, vol. 28, no. 3, pp. 363-374, 2007.

[15] D. Lukkassen, A. Meidell, and P. Wall, "Homogenization of some variational problems connected to the theory of lubrication," International Journal of Engineering Science, vol. 47, no. 1, pp. 153-162, 2009.

[16] D. Lukkassen, A. Meidell, and P. Wall, "Bounds on the effective behavior of a homogenized generalized Reynolds equation," Journal of Function Spaces and Applications, vol. 5, no. 2, pp. 133-150, 2007.

[17] A. Almqvist, E. K. Essel, J. Fabricius, and P. Wall, "Variational bounds applied to unstationary hydrodynamic lubrication," International Journal of Engineering Science, vol. 46, no. 9, pp. 891-906, 2008.

[18] A. Almqvist, D. Lukkassen, A. Meidell, and P. Wall, "New concepts of homogenization applied in rough surface hydrodynamic lubrication," International Journal of Engineering Science, vol. 45, no. 1, pp. 139-154, 2007. 
[19] N. Benhaboucha, M. Chambat, and I. Ciuperca, "Asymptotic behaviour of pressure and stresses in a thin film flow with a rough boundary," Quarterly of Applied Mathematics, vol. 63, no. 2, pp. 369-400, 2005.

[20] A. Almqvist, E. K. Essel, L. E. Persson, and P. Wall, "Homogenization of the unstationary incompressible Reynolds equation," Tribology International, vol. 40, no. 9, pp. 1344-1350, 2007.

[21] G. Bayada, I. Ciuperca, and M. Jai, "Homogenized elliptic equations and variational inequalities with oscillating parameters. Application to the study of thin flow behavior with rough surfaces," Nonlinear Analysis, vol. 7, no. 5, pp. 950-966, 2006. 


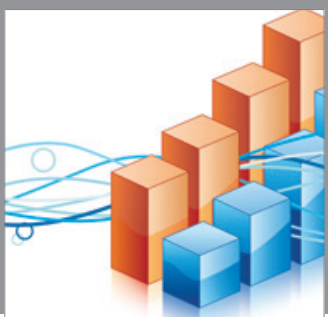

Advances in

Operations Research

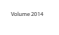

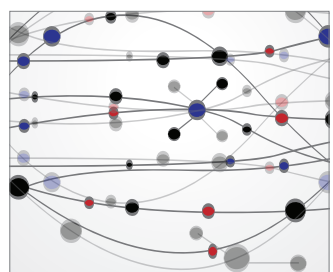

\section{The Scientific} World Journal
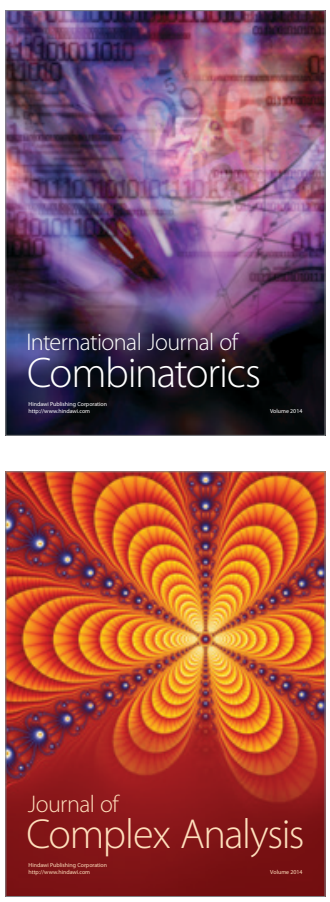

International Journal of

Mathematics and

Mathematical

Sciences
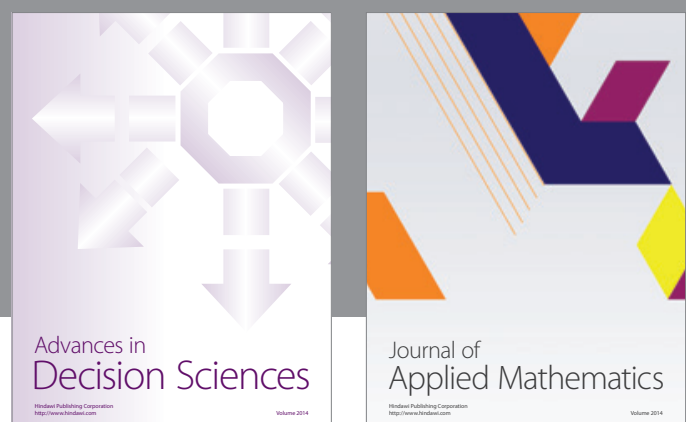

Journal of

Applied Mathematics
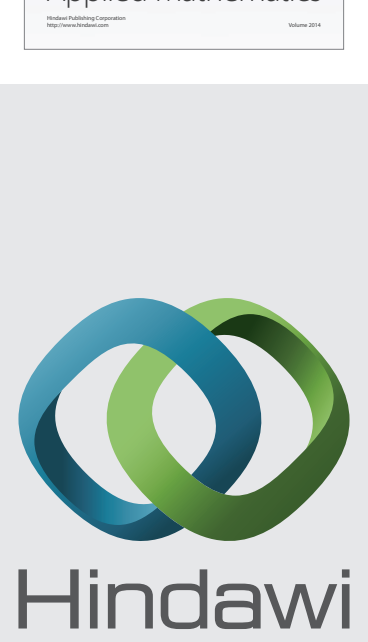

Submit your manuscripts at http://www.hindawi.com
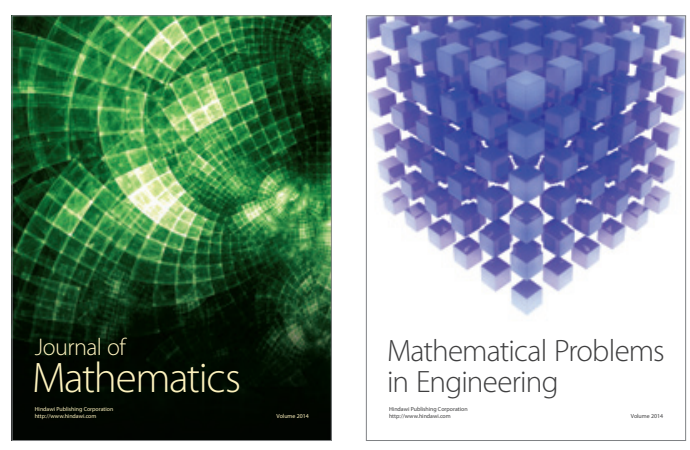

Mathematical Problems in Engineering
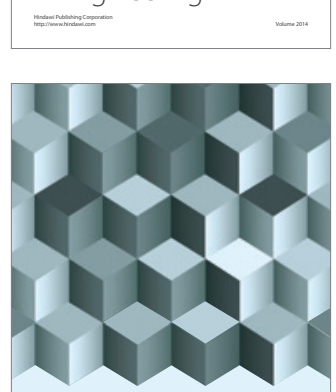

Journal of

Function Spaces
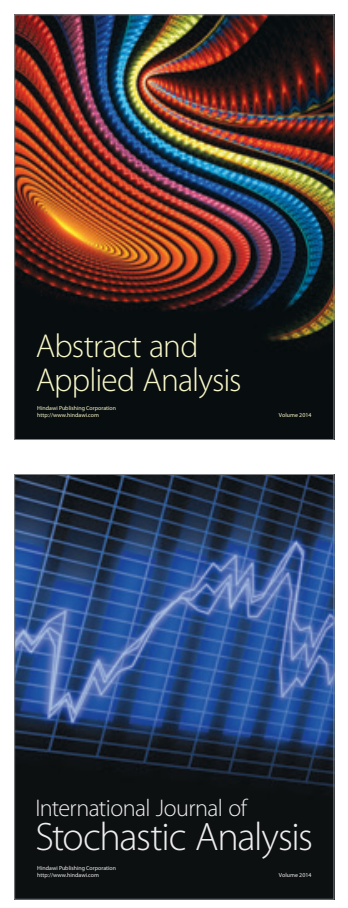

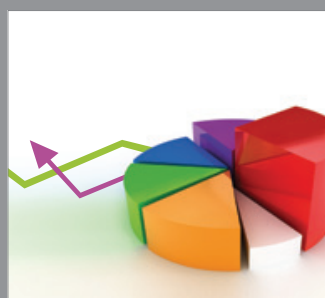

ournal of

Probability and Statistics

Promensencen
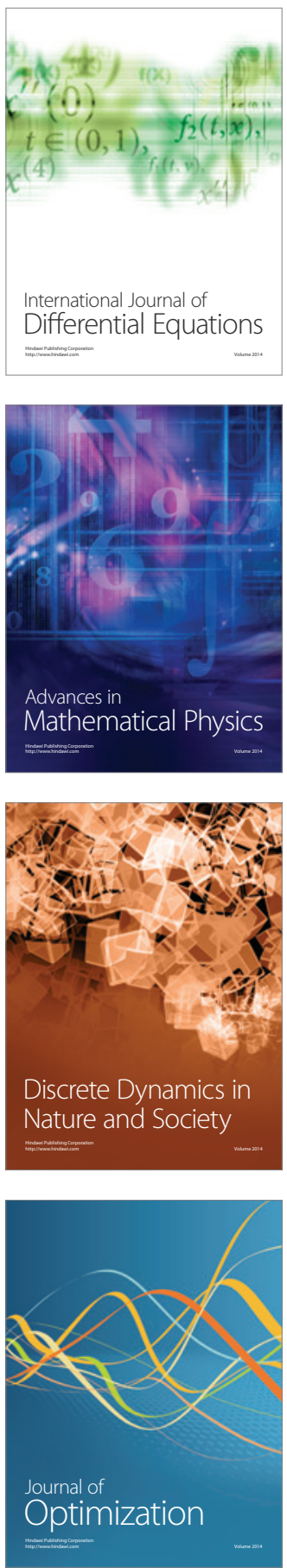